\title{
Pacific
}

Journal of

Mathematics

\section{INTERPOLATING BLASCHKE PRODUCTS}

Donald EdDy MARShall AND ARne STRAY

Volume 173 No. 2 


\title{
INTERPOLATING BLASCHKE PRODUCTS
}

\author{
Donald E. Marshall ANd ARne Stray
}

We prove that any bounded analytic function on the unit disk $\mathbb{D}$ which extends to be continuous on $\partial \mathbb{D} \backslash E$, for some set $E$ of measure 0 , can be uniformly approximated by finite linear combinations of interpolating Blaschke products.

Let $H^{\infty}$ denote the set of bounded analytic functions defined on the unit disk, $\mathbb{D}$. Each $f \in H^{\infty}$ has a non-tangential limit at almost all $e^{i \theta} \in \partial \mathbb{D}$ which we call $f\left(e^{i \theta}\right)$, and

$$
\sup _{z \in \mathbb{D}}|f(z)|=\|f\|_{\infty}
$$

where $\|f\|_{\infty}$ is the $L^{\infty} \equiv L^{\infty}(\partial \mathbb{D}, d \theta)$ norm of the non-tangential limit $f$. Thus we can view $H^{\infty}$ as a uniformly closed subalgebra of $L^{\infty}$. An inner function is a function $I \in H^{\infty}$ with $\left|I\left(e^{i \theta}\right)\right|=1$, a.e. $d \theta$. For example $S(z)=e^{\frac{z+1}{z-1}}$ is an inner function, though it has radial limit 0 at $\zeta=1$. Inner functions are of central importance in the study of analytic functions on $\mathbb{D}$. For example, Beurling used inner functions (and coined the name) to characterize the translation invariant subspaces of the Hardy space $H^{2}$. Rudin and Carleson used inner functions to characterize the ideals of the disk algebra, and Newman used them to characterize the Shilov boundary of $H^{\infty}$.

If $\left\{z_{n}\right\} \subset \mathbb{D}$ with $\sum_{n}\left(1-\left|z_{n}\right|\right)<\infty$ then a Blaschke product with zero set $\left\{z_{n}\right\}$ is an inner function of the form

$$
B(z)=\prod_{n=1}^{\infty} c_{n} \frac{z-z_{n}}{1-\overline{z_{n}} z}
$$

where $c_{n}$ are constants, with $\left|c_{n}\right|=1$, chosen so the the infinite product converges. Blaschke products are important because they characterize the zero sets of bounded analytic functions: if $f \in H^{\infty}$ then there is a Blaschke product $B$ and a non-vanishing $g \in H^{\infty}$ with $f=B g$ and $\left|f\left(e^{i \theta}\right)\right|=\left|g\left(e^{i \theta}\right)\right|$ almost everywhere on $\partial \mathbb{D}$. Frostman proved that if $I$ is inner then $(I-\lambda) /(1-$ $\bar{\lambda} I$ ) is a Blaschke product for $\lambda$ in a dense subset of $\mathbb{D}$ (the exceptional set in fact has logarithmic capacity 0 ). Letting $\lambda \rightarrow 0$ shows that every inner function can be uniformly approximated by Blaschke products. Because of 
their importance in this subject, the problem of characterizing the linear span of the inner functions arose in 1965. Douglas and Rudin proved that every unimodular function in $L^{\infty}$ can be uniformly approximated by the quotient of two inner functions. Since every $L^{\infty}$ function of norm smaller than 1 is the average of two unimodular functions, finite linear combinations of quotients of Blaschke products are dense in $L^{\infty}$. Later Hoffman proved that Blaschke products separated the points of the maximal ideal space of $H^{\infty}$. In [M1] it was proved that the unit ball of $H^{\infty}$ is the norm closed convex hull of the set of Blaschke products. See $[\mathbf{G}]$ and $[\mathbf{H}]$ for these and other background results about inner functions and Blaschke products.

Important developments in function theory on the disk have come from considering a special kind of zero set, called an interpolating sequence. The pseudo-hyperbolic metric on $\mathbb{D}$ is given by

$$
\rho(z, w)=\left|\frac{z-w}{1-\bar{w} z}\right| .
$$

A sequence $\left\{z_{n}\right\} \subset \mathbb{D}$ is called an interpolating sequence if

$$
\inf _{n} \prod_{j \neq n} \rho\left(z_{j}, z_{n}\right)>0 \text {. }
$$

An interpolating sequence is the zero set of a Blaschke product $B$ and the condition (1) can be rephrased in terms of $B$ since

$$
\left(1-\left|z_{n}\right|^{2}\right)\left|B^{\prime}\left(z_{n}\right)\right|=\prod_{j \neq n} \rho\left(z_{j}, z_{n}\right) .
$$

Such a Blaschke product is called an interpolating Blaschke product. Carleson proved that if $\left\{z_{n}\right\}$ is an interpolating sequence and if $\left\{a_{n}\right\} \in \ell^{\infty}$, then there is an $f \in H^{\infty}$ "interpolating" the values $a_{n}$ at $z_{n}$. In other words, $f\left(z_{n}\right)=a_{n}$ for all $n$. The formulation of interpolating sequences in terms of $B^{\prime}$ is important because the quantity $\left(1-|z|^{2}\right)\left|B^{\prime}(z)\right|$ is the conformally invariant derivative of $B$, in the following sense: Let $z=\tau(w)$ and $C(w)=$ $B(\tau(w))$, where $\tau$ is a conformal map of $\mathbb{D}$ onto $\mathbb{D}$. Then $\left(1-|z|^{2}\right)\left|B^{\prime}(z)\right|=$ $\left(1-|w|^{2}\right)\left|C^{\prime}(w)\right|$.

Carleson's proof of his interpolation theorem gave an important geometric characterization of interpolating sequences. A measure $\mu$ defined on $\mathbb{D}$ is called a Carleson measure if there is a constant $K<\infty$ so that for all approximate squares

$$
Q=\left\{r e^{i \theta}: 1-a<r<1, \theta_{0} \leq \theta \leq \theta_{0}+a\right\}
$$

the following estimate holds:

$$
\mu(Q) \leq K a
$$


A sequence $\left\{z_{n}\right\} \subset \mathbb{D}$ is interpolating if and only if there is an $\varepsilon>0$ such that

$$
\rho\left(z_{n}, z_{m}\right) \geq \varepsilon \text { for all } n \neq m \text { and }
$$

$$
\sum_{n}\left(1-\left|z_{n}\right|^{2}\right) \delta_{z_{n}} \text { is a Carleson measure, }
$$

where $\delta_{z}$ is unit point mass at $z$. Carleson measures and interpolating sequences also proved to be of great importance in the proof of the Corona theorem, by Carleson [Ca], and in finding $L^{\infty}$ solutions to $\bar{\partial}$-problems by Jones [J2], for example. Carleson measures, were also fundamental in the development of BMO by C. Fefferman [Fe], Jones [J3] and others. Carleson measures can be obtained as weak-* limits of measures associated with interpolating sequences [J3].

The problem of characterizing the linear span of the interpolating Blaschke products arose in 1978 [J1]. See also [G, p. 430]. Ziskind [Z] earlier proved that interpolating Blaschke products characterize the Shilov boundary of $H^{\infty}$, the natural analog of Newman's theorem. Another indication that the interpolating Blaschke products span a "large" subset of $H^{\infty}$ is the ChangMarshall theorem [Ch, M2]: Every closed subalgebra of $L^{\infty}$ containing $H^{\infty}$ is generated by $H^{\infty}$ and a collection of complex conjugates of interpolating Blaschke products. The analog of Frostman's theorem for interpolating Blaschke products is not true, however, since Kahane [K], Piranian [P], and Shapiro [S] proved there are inner functions $I$, which are not finite Blaschke products, such that

$$
\lim _{|z| \rightarrow 1}\left(1-|z|^{2}\right)\left|I^{\prime}(z)\right|=0 \text {. }
$$

Since $(I-\lambda) /(1-\bar{\lambda} I)$ must have this same property, it is never an interpolating Blaschke product. In [J1] Jones proved the analogs of the Douglas-Rudin and Hoffman theorems mentioned above: Every unimodular $L^{\infty}$ function can be uniformly approximated by a quotient of interpolating Blaschke products and the interpolating Blaschke products separate the points of the maximal ideal space of $H^{\infty}$.

Let $\mathcal{I} B P$ denote the uniform closure of finite linear combinations of interpolating Blaschke products:

$$
\mathcal{I} B P=c l\left\{\sum_{n=1}^{m} \alpha_{n} B_{n}: B_{n} \text { is interpolating and } \alpha_{n} \in \mathbb{C}\right\} .
$$

While we cannot solve Jones' problem of characterizing $\mathcal{I} B P$, we can prove that an $H^{\infty}$ function is in $\mathcal{I} B P$ if it is not too "pathological". Let $\mathcal{R} \mathcal{I}$ denote 
the bounded analytic functions on $D$ which extend to be continuous on on $\overline{\mathbb{D}} \backslash E$ for some $E \subset \partial \mathbb{D}$ with $|E| \equiv$ length $(E)=0$. These are precisely the bounded analytic functions with Riemann integrable boundary values, and hence the name $\mathcal{R I}$. By Corollary 1.3 of [DGG], $\mathcal{R} \mathcal{I}$ is the uniform closure of the set of bounded analytic functions that extend to be continuous on $\overline{\mathbb{D}} \backslash E$ for some closed set $E \subset \partial \mathbb{D}$ with $|E| \equiv$ length $(E)=0$.

\section{Theorem. $\mathcal{R} I \subset \mathcal{I} B P$.}

With different techniques, and at the same time, A. Nicolau proved that a Blaschke product which is continuous on $\overline{\mathbb{D}} \backslash E$ for some closed set $E \subset \partial \mathbb{D}$ with $|E| \equiv$ length $(E)=0$ is in the algebra generated by the interpolating Blaschke products. We remark that Wolff [unpublished] has shown that there are Blaschke products in $\mathcal{R} \mathcal{I}$ satisfying (5) above.

Lemma 1. IBP is an algebra.

Proof of Lemma 1. It suffices to show that the product of two interpolating Blaschke products is in $\mathcal{I} B P$. Suppose $B_{1}$ and $B_{2}$ are interpolating Blaschke products with zero sets $\left\{a_{n}\right\}$ and $\left\{b_{n}\right\}$ respectively, then $\left\{z_{n}\right\}=\left\{a_{n}\right\} \cup\left\{b_{n}\right\}$ satisfies (4). It is possible to move the $\left\{b_{n}\right\}$ slightly so as to satisfy (3) and still have a Blaschke product close to $B_{1} B_{2}$. The $\left\{b_{n}\right\}$ cannot just be arbitrarily moved, for example if $a_{n}=b_{n}=1-2^{-n}$ and if $B_{3}$ is the Blaschke product with zeros $\zeta_{n}=z_{n}+i \delta 2^{-n}$, where $\delta>0$, then $\left\|B_{1} B_{2}-B_{1} B_{3}\right\|=2$.

By (3), we may suppose that the disks $D_{k}=\left\{z: \rho\left(z, a_{k}\right) \leq \delta\right\}$ are disjoint and each $D_{k}$ contains at most one $b_{n}$. By Earl's proof of Carleson's interpolation theorem [E], if $\delta$ is sufficiently small, there is a constant $K$, $0<K<1$ so that

$$
\left|B_{1}(z)\right| \geq K \delta
$$

on $\Omega_{\delta}=\mathbb{D} \backslash \cup_{k} D_{k}$. Moreover, we can move some of the zeros of $B_{1}$ to $B_{2}$ so that we can assume that for each zero $a_{k}$ of $B_{1}$, there is a zero $d_{k}$ of $B_{2}$ with $\rho\left(a_{k}, d_{k}\right)<K \delta / 4$. By Frostman's theorem, we can choose $\varepsilon$, with $K \delta / 4<$ $\varepsilon<K \delta / 3$ so that $C_{3}=\left(B_{1}-\varepsilon\right) /\left(1-\varepsilon B_{1}\right)$ is a Blaschke product. Then $C_{3}$ has exactly one zero in each "annular" region $A_{k}=\left\{z: \varepsilon<\rho\left(z, a_{k}\right)<\delta\right\}$ and no other zeros. To see this, first note that

$$
\left\|C_{3}-B_{1}\right\|_{\infty} \leq \frac{2 \varepsilon}{1-\varepsilon}<K \delta .
$$

So if $C_{3}(z)=0$, then $\left|B_{1}(z)\right|<K \delta$ and hence by (6) $z \notin \Omega_{\delta}$. Also if $C_{3}(z)=0$, then

$$
\varepsilon=\left|B_{1}(z)\right|=\rho\left(B_{1}(z), B_{1}\left(a_{n}\right)\right)<\rho\left(z, a_{n}\right)
$$


by Schwarz's lemma. On $\partial D_{k}$, by (6) and (7),

$$
\left|C_{3}(z)-B_{1}(z)\right|<\left|B_{1}(z)\right|
$$

so Rouché's theorem, $C_{3}$ has exactly one zero in each $D_{k}$ and hence exactly one in each $A_{k}$. Thus $\rho$-distance between the zeros of $C_{3}$ and the zeros of $B_{2}$ is at least $\rho(\varepsilon, K \delta / 4)>0$. It is not hard to verify, using (3) and (4), that $B \equiv C_{3} B_{2}$ is interpolating and

$$
\left\|B_{1} B_{2}-B\right\|_{\infty}<\frac{2 \varepsilon}{1-\varepsilon}
$$

Since $\delta$, and hence $\varepsilon$, can be chosen arbitrarily small, this proves the lemma.

We remark that since $z \in \mathcal{I} B P$

$$
1=-2\left(\frac{z-\frac{1}{2}}{1-\frac{1}{2} z}\right)+3 \sum_{n=1}^{\infty} \frac{z^{n}}{2^{n}} \in \mathcal{I} B P,
$$

by Lemma 1 .

The estimate in the next Lemma is the key to the proof of the theorem. We transfer the problem from the disk to the upper half plane $\mathbb{H}$. In this context, the pseudo-hyperbolic distance is given by

$$
\rho(z, w)=\left|\frac{z-w}{z-\bar{w}}\right|
$$

$z, w \in \mathbb{H}$, and a Blaschke product is given by

$$
B(z)=\prod_{n=1}^{\infty} c_{n} \frac{z-z_{n}}{z-\bar{z}_{n}}
$$

where $\operatorname{Im} z_{n}>0$, and $\left\{c_{n}\right\}$ are constants of absolute value 1 chosen so that the product converges. The conformally invariant derivative of $B$ on the upper half plane is $\operatorname{Im} z\left|B^{\prime}(z)\right|$, and $B$ is interpolating if and only if

$$
\inf _{n} 2 \operatorname{Im} z_{n}\left|B^{\prime}\left(z_{n}\right)\right|=\inf _{m} \prod_{n \neq m} \rho\left(z_{n}, z_{m}\right)>0 .
$$

Lemma 2. Suppose $B$ is a Blaschke product on $\mathbb{H}$ with zeros $\left\{z_{n}\right\} \subset \mathbb{H} \cap\{z$ : $\operatorname{Re} z \leq 0\}$. If $\delta>0$ and $0<\arg z<\frac{\pi}{2}-\delta$, then

$$
\operatorname{Im} z\left|B^{\prime}(z)\right| \geq C|B(z)| \log \frac{1}{|B(z)|}
$$


where $C$ is a constant that depends only on $\delta$

Proof of Lemma 2. Note that for some $\varepsilon=\varepsilon(\delta)>0, \rho\left(z, z_{n}\right) \geq \varepsilon$. Thus

$$
\log \frac{1}{|B(z)|^{2}} \leq C_{1} \sum_{n=1}^{\infty}\left(1-\rho^{2}\left(z, z_{n}\right)\right)=4 C_{1} \sum_{n=1}^{\infty} \frac{\operatorname{Im} z \operatorname{Im} z_{n}}{\left|z-\bar{z}_{n}\right|^{2}},
$$

and

$$
(\operatorname{Im} z) \frac{B^{\prime}(z)}{B(z)}=\sum_{n=1}^{\infty} \frac{\operatorname{Im} z \operatorname{Im} z_{n}}{\left|z-\bar{z}_{n}\right|^{2}} \frac{2 i\left(\bar{z}-z_{n}\right)}{z-z_{n}} .
$$

By our assumptions on $z$ and $z_{n}$, (see Figure 1)

$$
0 \leq \theta=\arg \left(\frac{z-z_{n}}{\bar{z}-z_{n}}\right) \leq \pi-2 \delta .
$$

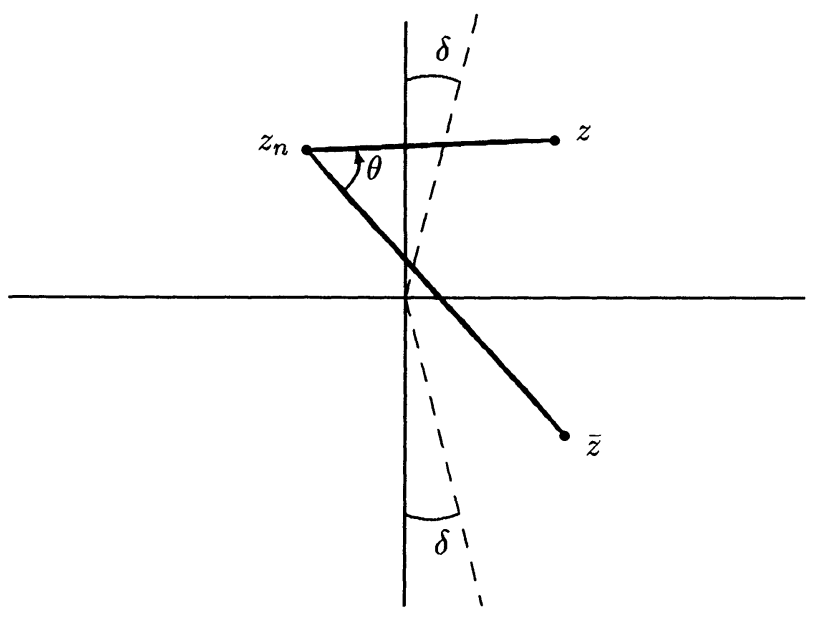

Figure 1.

Thus if $\zeta=e^{-i \delta}$,

$$
\left|\arg \left(\frac{2 i\left(\bar{z}-z_{n}\right)}{z-z_{n}} \zeta\right)\right| \leq \frac{\pi}{2}-\delta
$$

and since $\left|\frac{\bar{z}-z_{n}}{z-z_{n}}\right| \geq 1$,

$$
\operatorname{Re} \frac{2 i\left(\bar{z}-z_{n}\right)}{z-z_{n}} \zeta \geq C_{2} \equiv 2 \cos \left(\frac{\pi}{2}-\delta\right)>0 .
$$

By (9), (10) and (11)

$\operatorname{Im} z\left|\frac{B^{\prime}(z)}{B(z)}\right| \geq \operatorname{Im} z \operatorname{Re}\left(\frac{B^{\prime}(z) \zeta}{B(z)}\right) \geq C_{2} \sum_{n=1}^{\infty} \frac{\operatorname{Im} z \operatorname{Im} z_{n}}{\left|z-\bar{z}_{n}\right|^{2}} \geq \frac{C_{2}}{4 C_{1}} \log \frac{1}{|B(z)|^{2}} . \square$ 
Lemma 3. If $B$ is a Blaschke product which is continuous on $\partial \mathbb{D} \backslash E$, where $E$ is a closed set of length 0 , then $B \in \mathcal{I} B P$.

Proof of Lemma 3. Transfer the problem to the upper-half plane $\mathbb{H}$ and without loss of generality, suppose the zeros $\left\{z_{n}\right\}$ of

$$
B(z)=\prod_{n=1}^{\infty} c_{n} \frac{z-z_{n}}{z-\bar{z}_{n}}
$$

are contained in the unit square $Q_{0}=\{x+i y: 0<x<1,0<y<1\}$. A dyadic square $Q$ is a square sitting on $\mathbb{R}$ of the form $Q=\left\{x+i y: \frac{k}{2^{n}} \leq x \leq\right.$ $\left.\frac{k+1}{2^{n}}, 0<y \leq 2^{-n}\right\}$ where $k$ and $n$ are integers. Let $\left\{Q_{j}\right\}$ be the set of dyadic squares which do not contain any zero of $B$. The region $Q_{0} \backslash \cup Q_{j}$ contains all zeros of $B$ and by our assumption on $B$, the curve $\Gamma=\partial\left(Q_{0} \backslash \cup Q_{j}\right)$ satisfies

$$
\text { length }(\Gamma \cap \mathbb{R})=0 \text {. }
$$

Note that arc-length on $\Gamma$ is a Carleson measure. Given $\varepsilon>0$, sprinkle points $\left\{\zeta_{n}\right\}$ on $\Gamma$ such that

$$
\rho\left(\zeta_{n}, \zeta_{m}\right) \geq \varepsilon \text { for all } n \neq m \text { and }
$$$$
\text { if } \zeta \in \Gamma \cap \mathbb{H} \text { then } \rho\left(\zeta, \zeta_{n}\right) \leq 2 \varepsilon, \text { for some } n \text {. }
$$

As in Ziskind [Z] (see also [G]), the Blaschke product $B_{I}$ with zeros $\left\{\zeta_{n}\right\}$ is interpolating. Moreover

$$
\left|B B_{I}\right| \leq 2 \varepsilon \text { on } Q_{0} \backslash \cup Q_{j}
$$

by (12), (14) and the maximum principle.

There is a $c_{1}, 0<c_{1}<1$, so that if $\rho\left(z, Q_{0} \backslash \cup Q_{j}\right)>c_{1}$ and $z \in Q_{0}$, then $z$ lies in a disk $D$ centered on $\mathbb{R}$ with $D \subset \cup Q_{j}$. Thus there is a $\sigma>2 \varepsilon$ and $\delta>0$, so that if $\left|B(z) B_{I}(z)\right|>\sigma$ then there is a linear fractional transformation $\varphi$ of $\mathbb{H}$ onto $\mathbb{H}$ so that $\varphi\left(Q_{0} \backslash \cup Q_{j}\right) \subset\{z: \operatorname{Re} z \leq 0\}$ and $0<\arg \varphi(z)<\frac{\pi}{2}-\delta$. A similar argument works for $z \notin Q_{0}$.

By (8), Lemma 2 and Frostman's theorem

$$
D=\frac{B B_{I}-\lambda}{1-\lambda B B_{I}}
$$

is an interpolating Blaschke product for some $\lambda>\sigma$. By Lemma 1 and the comment immediately after Lemma 1 ,

$$
B B_{I}=\lambda+\left(1-\lambda^{2}\right) \sum_{n=1}^{\infty}(-\lambda)^{n-1} D^{n} \in \mathcal{I} B P .
$$


By the arguments used in the proof of Lemma 1 and Frostman's theorem,

$$
\frac{B_{I}-\eta}{1-\bar{\eta} B_{I}}
$$

is an interpolating Blaschke product for some arbitrarily small $|\eta|$. In the proof above that $D$ is interpolating, we really only needed that $B_{I}$ is small on $\Gamma$ and that the zeros of $B_{I}$ are hyperbolically close to $\Gamma$. Thus

$$
C=B\left(\frac{B_{I}-\eta}{1-\bar{\eta} B_{I}}\right) \in \mathcal{I} B P .
$$

and

$$
B=\frac{1}{\eta}\left(B B_{I}-C\left(1-\bar{\eta} B_{I}\right)\right) \in \mathcal{I} B P .
$$

Proof of the Theorem. By the comment after the definition of $\mathcal{R} \mathcal{I}$ and since $1 \in \mathcal{I} B P$, it suffices to approximate only $f \in H^{\infty}$ such that $f$ extends to be continuous on $\overline{\mathbb{D}} \backslash E$, where $E$ is closed and $|E|=0$, and such that $\left\|f-\frac{1}{2}\right\|_{\infty}<\frac{1}{2}$. Let $C_{E}$ denote the closed subalgebra of $L^{\infty}$ generated by quotients of Blaschke products which extend to be analytic on $\partial \mathbb{D} \backslash E$. In other words, $C_{E}$ is the set of uniform limits of finite linear combinations of quotients of Blaschke products which are analytic on $\partial \mathbb{D} \backslash E$. Since each point of $\left\{z:\left|z-\frac{1}{2}\right|<\frac{1}{2}\right\}$ is the average of two unique points on $\partial \mathbb{D}$, we can write $f=\left(u_{1}+u_{2}\right) / 2$ where $u_{1}$ and $u_{2}$ are unimodular functions on $\partial \mathbb{D}$ which are continuous on $\partial \mathbb{D} \backslash E$. By Theorem 12.1 [DDG], we have $f \in C_{E}$. Let $\mathcal{A}$ be the uniformly closed algebra generated by $H^{\infty}$ and $C_{E}$. It is shown in [MS] that the inner functions which are invertible in $\mathcal{A}$ belong to $C_{E}$. By Theorem 4.1 [CM], $f$ can be uniformly approximated by convex combinations of Blaschke products in $H^{\infty} \cap C_{E}$. The Theorem now follows from Lemma 1 and Lemma 3.

We remark that $\mathcal{R} \mathcal{I}$ is a uniformly closed subalgebra of $H^{\infty}$ with innerouter factorization. See [MS].

\section{References}

[Ca] L. Carleson, Interpolations by bounded analytic functions and the corona problem, Annals of Math., 76 (1962), 547-559.

[Ch] S.Y. Chang, A characterization of Douglas subalgebras, Acta Math., 137 (1976), 81-89.

[CM] S.Y. Chang and D.E. Marshall, Some algebras of bounded analytic functions containing the disk algebra, Banach Spaces of Analytic Functions, Springer Lecture Notes, 604 (1977), 12-20. 
[DGG] A.M. Davie, T.W. Gamelin, and J. Garnett, Distance estimates and pointwise bounded density, Trans. Amer. Math. Soc., 175 (1973), 37-68.

[E] J.P. Earl, On the interpolation of bounded sequences by bounded functions, J. London. Math. Soc., 2 (1970), 544-548.

[Fe] C. Fefferman and E.M. Stein, $H^{p}$ spaces of several variables, Acta Math., 129 (1972), 137-193.

[Fr] O. Frostman, Potentiel d'équilibre et capacité des ensembles avec quelques applications á la théorie des fonctions, Medd. Lund. Univ. Math. Sem., 3 (3) (1935), 1-118.

[G] J.B. Garnett, Bounded Analytic Functions, Academic Press, New York, 1981.

[H] K. Hoffman, Banach Spaces of Analytic Functions, Prentiss-Hall, Inc., Englewood Cliffs, 1962.

[J1] P.W. Jones, Constructions with Functions of Bounded Mean Oscillation, Thesis, UCLA, 1978. See also P.W. Jones, Ratios of interpolating Blaschke products, Pac. J. Math., 95 (1981), 311-321.

[J2] $\longrightarrow L^{\infty}$ estimates and the $\partial$-problem, Acta Math., 150 (1983), 137-152.

[J3] Carleson measures and the Fefferman-Stein decomposition of $\mathrm{BMO}(\mathbb{R})$, Annals of Math., 111 (1980), 197-208.

[K] J.P. Kahane, Trois notes sur les esembles parfaits linéaires, Enseign. Math., 15 (1969), 185-192.

[M1] D.E. Marshall, Blaschke products generate $H^{\infty}$, Bull. Amer. Math. Soc., 82 (1976), 494-496.

[M2] - Subalgebras of $L^{\infty}$ containing $H^{\infty}$, Acta Math., 137 (1976), 91-98.

[MS] D.E. Marshall and K. Stephenson, Inner divisors and composition operators, J. Funct. Anal., 46 (1982), 131-148.

[P] G. Piranian, Two monotonic singular, uniformly almost smooth functions, Duke Math J., 33 (1966), 255-262.

[S] H.S. Shapiro, Monotonic singular functions of high smoothness, Michigan Math. J., 15 (1968), 265-275.

[Z] S. Ziskind, Interpolating sequences and the Shilov boundary of $H^{\infty}(\Delta)$, J. Funct. Anal., 21 (1976), 380-388.

Received August 10, 1993. The first author is supported in part by NSF grant DMS9302823.

UNIVERSITY OF WASHINGTON

SEATTLE, WA 98195

E-mail address: marshall@math.washington.edu

AND

UNIVERSITETET I BERGEN

MATEMATISK INSTITUTT

5014 BERGEN-UNIVERSITETET

NORWAY 

Peng Lin and Richard Rochberg, Trace ideal criteria for Toeplitz and Hankel operators on the weighted Bergman spaces with exponential type

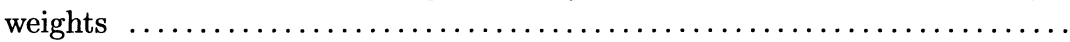

Donald E. Marshall and Arne Stray, Interpolating Blaschke products . .

Kathy D. Merrill and Lynne $\mathbf{H}$. Walling, On quadratic reciprocity over

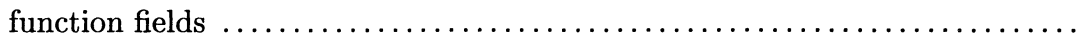

Takahiko Nakazi and Masahiro Yamada, $\left(A_{2}\right)$-conditions and Carleson

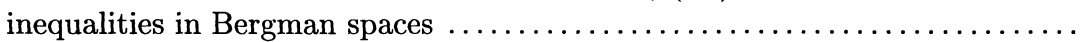

C. Ott, A note on a paper of E. Boasso and A. Larotonda ..............

Victor Patrangenaru, Classifying 3 and 4 dimensional homogeneous Riemannian manifolds by Cartan triples

Carlo Pensavalle and Tim Steger, Tensor products with anisotropic principal series representations of free groups

Ying Shen, On Ricci deformation of a Riemannian metric on manifold with boundary

Albert Jeu-Liang Sheu, The Weyl quantization of Poisson $S U(2) \ldots \ldots$

Alexandra Shlapentokh, Polynomials with a given discriminant over fields of algebraic functions of positive characteristic

Eric Stade and D.I. Wallace, Weyl's law for $S L(3, \mathbb{Z}) \backslash S L(3, \mathbb{R}) / S O(3, \mathbb{R})$

Christopher W. Stark, Resolutions modeled on ternary trees ........ 557

Per Tomter, Minimal hyperspheres in two-point homogeneous spaces .....

Jun Tomiyama, Topological Full groups and structure of normalizers in

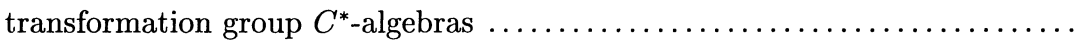

Nik Weaver, Subalgebras of little Lipschitz algebras 


\section{PACIFIC JOURNAL OF MATHEMATICS}

\section{Volume $173 \quad$ No. $2 \quad$ April 1996}

A mean value inequality with applications to Bergman space operators

PATRICK ROBERT AHERn and ZELJKO CUCKOVIC

$H^{p}$-estimates of holomorphic division formulas

MATS ANDERSSON and HASSE CARLSSON

Group structure and maximal division for cubic recursions with a double root

Christian JeAn-Claude Ballot

The Weil representation and Gauss sums

ANTONiA WiLson BLUHER

Duality for the quantum $E(2)$ group

ALFONS VAN DAELE and S. L. WORONOWICZ

Cohomology complex projective space with degree one codimension-two fixed submanifolds 387

KARL HEINZ DOVERMANN and ROBERT D. LITTLE

On the mapping intersection problem

ALEXANDER DRANISHNIKOV

From the $L^{1}$ norms of the complex heat kernels to a Hörmander multiplier theorem for

sub-Laplacians on nilpotent Lie groups

\section{XUAN THINH DUONG}

Isoperimetric inequalities for automorphism groups of free groups

Allen E. Hatcher and Karen Vogtmann

Approximation by normal elements with finite spectra in $C^{*}$-algebras of real rank zero

HUAXIN LIN

Interpolating Blaschke products

DonALD EdDY MARShall and ARNE STRAY

Interpolating Blaschke products generate $H^{\infty}$

JOHN BRADY GARNETT and ARTUR NICOLAU

Classifying 3- and 4-dimensional homogeneous Riemannian manifolds by Cartan triples

VICTOR PATRANGENARU

Polynomials with a given discriminant over fields of algebraic functions of positive

characteristic

ALEXANDRA SHLAPENTOKH

Resolutions modeled on ternary trees

CHRISTOPHER W. STARK

Topological full groups and structure of normalizers in transformation group $C^{*}$-algebras 